\title{
Evaluación energética y exergética en un motor de combustión interna ciclo Otto de 1.6L
}

\section{(Energy and exergy evaluation in a 1.6L Otto cycle internal combustion engine)}

\author{
Edilberto Antonio Llanes-Cedeño ${ }^{1}$, José Bolívar Carguachi-Caizatoa ${ }^{1,2}$, Juan Carlos Rocha- \\ Hoyos $^{1}$
}

\begin{abstract}
Resumen:
El presente trabajo tuvo como objetivo evaluar el comportamiento de un motor de combustión interna de ciclo Otto mediante el balance de energía y exergía para la valorización de su rendimiento; se consideró como caso de estudio un motor de $1.6 \mathrm{~L}$. El cálculo energético se desarrolló en una ruta previamente caracterizada a una velocidad constante de $50 \mathrm{~km} / \mathrm{h}$ y $90 \mathrm{~km} / \mathrm{h}$. Tras una investigación de tipo experimental, con base en observación y medición, además de métodos de inducción, deducción y síntesis, se determinó, dentro de las principales conclusiones, que el análisis del balance energético y exergético permite conocer el rendimiento real de un motor de combustión interna. Mediante este análisis, se determinó que el motor estudiado presenta un rendimiento energético promedio de $27.57 \%$ para una velocidad de $50 \mathrm{~km} / \mathrm{h}$, mientras que el flujo de exergía total del sistema es $22 \%$, no existiendo diferencia significativa con los resultados de eficiencia a $90 \mathrm{~km} / \mathrm{h}$.
\end{abstract}

Palabras clave: motor de combustión interna; balance energético; balance exergético.

\begin{abstract}
:
This paper aimed to evaluate the behavior of an Otto Cycle internal combustion of a $1.6 \mathrm{~L}$ engine measuring its performance by the energy and exergy balance. The energy calculation was developed in a previously set route at a constant speed of $50 \mathrm{~km} / \mathrm{h}$ and $90 \mathrm{~km} / \mathrm{h}$. It was determined, that the analysis of the energy and exergy balance contributes to recognize the performance of an internal combustion after the experimentation based on observation, measurement, methods of induction, deduction and synthesis. Also, it was resolved that the engine has an average energy efficiency of $27.57 \%$ for a speed of $50 \mathrm{~km} / \mathrm{h}$, while the total exergy flow of the system is $22 \%$. Therefore, there is no significant difference with the efficiency results at $90 \mathrm{~km} / \mathrm{h}$.
\end{abstract}

Keywords: internal combustion engine; energy balance; exergy balance.

\section{Introducción}

Los motores de combustión interna (MCl), junto con el uso cotidiano de la electricidad, determinaron un hito que marcó la historia de la industria y la sociedad a finales del siglo XIX y principios del siglo XX. El uso de los $\mathrm{MCl}$ revolucionó el transporte y su uso se extendió de manera progresiva y provocó un incremento en la movilidad de las personas y los productos, la cual facilitó la producción y el comercio, y por ende el estándar de vida, además de los avances técnicos que se han logrado en la actualidad (Vásquez et al., 2004; Llanes et al., 2017).

A pesar de los beneficios que ha supuesto el desarrollo de los $\mathrm{MCl}$, también se deben considerar los costos, especialmente en el tema ambiental por el uso de combustibles fósiles. El cambio climático, uno de los problemas de mayor investigación en la actualidad, está

\footnotetext{
${ }^{1}$ Universidad Internacional SEK, Quito, Ecuador (\{antonio.llanes, carlos.rocha, jcarguachi.mec\} @uisek.edu.ec).

2 Instituto Tecnológico Superior Sucre, Quito, Ecuador (jcarguachi@tecnologicosucre.edu.ec).
} 
asociada a la utilización de estos combustibles desde los inicios de la era industrial (Duarte, 2006; Rocha et al., 2018).

Un mundo más sostenible requiere de la implementación de equipos de uso de hidrocarburos de alta eficiencia, sistemas y procesos para reducir las emisiones de gases de efecto invernadero y conservación de los combustibles fósiles. La producción de electricidad y transporte es una de las principales causas de los actuales niveles de emisiones de gases de efecto invernadero (Segura \& Arriaga, 2003; Santo, 2012).

La gasolina Extra en Ecuador, que es la más utilizada con alrededor de $89 \%$ del consumo de vehículos a gasolina (López, 2013; Erazo, 2015), tiene un octanaje de 87, mientras que la gasolina Súper, con $11 \%$ del consumo, tiene 92 octanos. El octanaje determina que tan fácil o difícil es que una gasolina explote dentro del cilindro del motor de un vehículo al ser sometido a compresión (Andrade, 2016). A mayor octanaje, aumenta la capacidad antidetonante del combustible, lo que mejora su eficiencia cuando se incrementa la relación volumétrica y previene daños en el motor.

El desarrollo de los $\mathrm{MCI}$ no se ha detenido. Durante las pasadas décadas, han surgido nuevos factores que afectan significativamente el diseño y desempeño del motor, entre los que destacan el control de las emisiones contaminantes y la reducción del consumo de combustible (Cedeño et al., 2017). En la actualidad se realizan muchas investigaciones con el fin de mejorar no solo los motores de combustión interna sino los combustibles utilizados (Cedeño, Rocha-Hoyos, Zurita, y Milla, 2018). Las investigaciones y avances incluyen el uso de gas natural, etanol, metanol, combustibles no derivados del petróleo (de origen vegetal), la gasolina sintética y el hidrógeno (Gaviria et al., 2002).

Dados los problemas de la energía y el ambiente que son cada vez más graves, la mejora del proceso de combustión en el cilindro no puede cumplir los requerimientos de mayor eficiencia térmica de los $\mathrm{MCl}$ por sí sola. Actualmente, muchas investigaciones apuntan a la recuperación de calor (residuos) de gas y agua de refrigeración para mejorar la economía de combustible y la reducción de las emisiones de $\mathrm{CO}_{2}$ (Zhu et al., 2014).

El análisis del balance energético de un $\mathrm{MCl}$, también denominado balance térmico o balance de calor, es básicamente una aplicación de la primera ley de la Termodinámica (Abedin et al., 2013). La eficiencia energética y exergética de los motores es un tema de singular importancia; aunque teóricamente esa eficiencia podría llegar hasta un $56.5 \%$ en condiciones ideales, los $\mathrm{MCl}$ tienen porcentajes de rendimiento (calor que se transforma efectivamente en trabajo) muy inferiores como se muestra en la Figura 1.

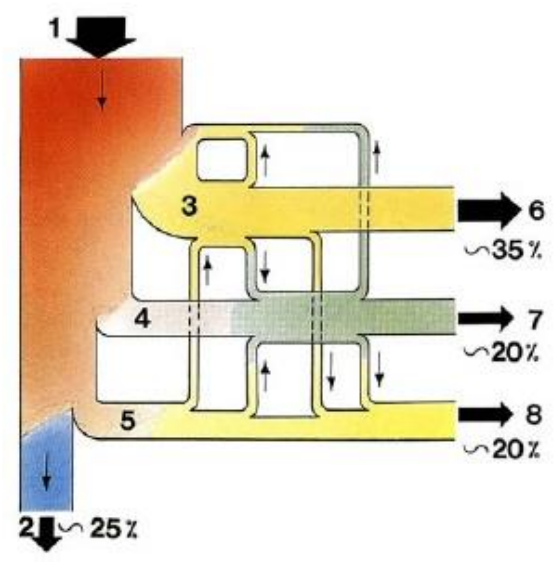

1. Poder calorífico del combustible.

2. Calor transformado en trabajo.

3. Calor contenido en los gases de escape.

4. Calor transmitido por los gases a las paredes.

5. Calor dado por las resistencias pasivas.

6. Calor perdido con los gases de escape.

7. Calor perdido en el líquido refrigerante.

8. Calor perdido por irradiación.

Figura 1. Balance térmico del motor de combustión interna. Fuente: Grupo FIAT (2004)

Por lo expresado anteriormente, el trabajo tiene como objetivo: evaluar el comportamiento de un motor de combustión interna de ciclo Otto mediante el balance de energía y exergía para la valorización de su rendimiento. 


\section{Metodología}

En un motor, considerándolo desde el punto de vista termodinámico, el sistema está comprendido por el gas atrapado en la cámara de combustión durante la compresión, la combustión y la expansión, como se muestra en la Figura 2. Este gas es una mezcla de combustible, aire, gases residuales y gases de escape recirculados. Tras la combustión, los reactantes se convierten en productos como $\mathrm{CO}_{2}, \mathrm{H}_{2} \mathrm{O}$, CO, etc., mientras que la energía química se convierte en energía térmica. La Figura 2, muestra que las paredes de la cámara de combustión constituyen fronteras del sistema. Aplicando la Primera Ley de la Termodinámica, la Ecuación 1 que acompaña a la Figura 2, equivale a decir que "la variación de la energía del sistema $\left(E_{s i s}\right)$, asumiendo que no hay pérdidas de energía en deformaciones del volumen de control, se reduce a la variación de la energía interna del sistema $(U)$ " (Piedrahita, 2009).

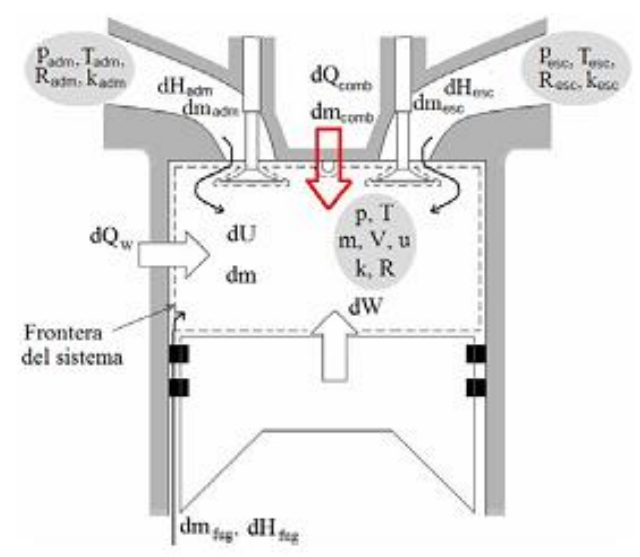

1. Energía del sistema (Esis)

2. Energía suministrada al sistema con el combustible (Qcomb)

3. Carga de admisión ( $\left.m a d m^{*} h a d m\right)$

4. Trabajo mecánico $(W)$

5. Calor disipado a través del refrigerante (Qrefr)

6. Calor de los gases de escape (mesc $\left.{ }^{*} h e s c\right)$

7. Calor equivalente de los gases de la cámara que se fugan a través de intersticios entre los segmentos (mfug*hfug).

Figura 2. Modelo termodinámico del motor de combustión interna Fuente: Piedrahita (2009)

$$
\frac{d E_{s i s}}{d t}=\frac{d U}{d t}=\frac{d Q_{c o m b}}{d t}+\frac{d Q_{r e f r}}{d t}+\frac{d W}{d t}+\frac{d m_{a d m}}{d t} h_{a d m}+\frac{d m_{e s c}}{d t} h_{e s c}+\frac{d m_{f u g}}{d t} h_{f u g}
$$

En lo referente a su alcance, esta investigación es de tipo experimental pues estudia el problema de una manera controlada para la obtención de resultados y su posterior análisis y discusión (Bernal, 2010). El vehículo que se somete a estudio es un auto liviano a gasolina con cinco asientos, con una longitud de 4.46 metros y un peso especificado de fábrica de $1270 \mathrm{~kg}$. El motor es de 1.6 litros de cilindrada, de 4 cilindros en línea y las especificaciones indican una potencia de $77 \mathrm{~kW}$ a $6000 \mathrm{rpm}$.

Para la realización del estudio, se utilizaron los siguientes materiales e instrumentos:

- Vehículo a gasolina 1.6 L, como $\mathrm{MCl}$ de estudio

- Escáner portátil OBD2 ELM 327

- Termómetro infrarrojo modelo DT 8000.

El experimento siguió los pasos que se describen a continuación:

- Puesta en marcha del motor hasta alcanzar la temperatura nominal de trabajo $90^{\circ} \mathrm{C}$.

- Aceleración del motor a velocidades $50 \mathrm{~km} / \mathrm{h}$ y 90 km/h a $2500 \mathrm{rpm}$ y $3300 \mathrm{rpm}$ respectivamente, las cuales serán las condiciones requeridas para el desarrollo del análisis del Balance Térmico en el MCl.

- Esperar hasta que la velocidad se estabilice (velocidad constante). 
- Los parámetros de medición se los realiza cada un minuto por diez minutos consecutivos.

- Con el motor apagado, se realiza la medición de temperaturas y área físicas en el escape del motor y cárter, esto ayudará a calcular las pérdidas por calor.

Para la elaboración del análisis térmico del $\mathrm{MCl}$, se realizó la adquisición de datos requeridos bajo condiciones controladas del vehículo en movimiento a una velocidad constante de $50 \mathrm{~km} / \mathrm{h}$ (2 $500 \mathrm{rpm}$ ) y $90 \mathrm{~km} / \mathrm{h}$ (3 $300 \mathrm{rpm}$ ), con ayuda del escáner ELM 327 y la aplicación Android.

Para tabular los datos se realizaron pruebas por 5 días; diariamente se registró los resultados durante diez minutos, para al final, sacar un promedio de los valores adquiridos. Esto se desarrolló en condiciones controladas tanto para la velocidad como para las revoluciones. Para poder mantener dichas condiciones, las pruebas se realizaron en el autódromo "José Tobar", de la cuidad de lbarra, el cual rodea la laguna de Yaguarcocha; este se ubica a 2 horas al norte de la ciudad de Quito, Ecuador, en las coordenadas $0^{\circ} 22^{\prime} 48^{\prime \prime} \mathrm{N}$ $78^{\circ} 5^{\prime} 48$ "W, con un área de $4097 \mathrm{~m}^{2}$, la pista mide $10 \mathrm{~km}$ de largo y 11 metros de ancho, a una altura promedio de 2220 m.s.n.m. En la Figura 3 se ilustra el área de la experimentación.

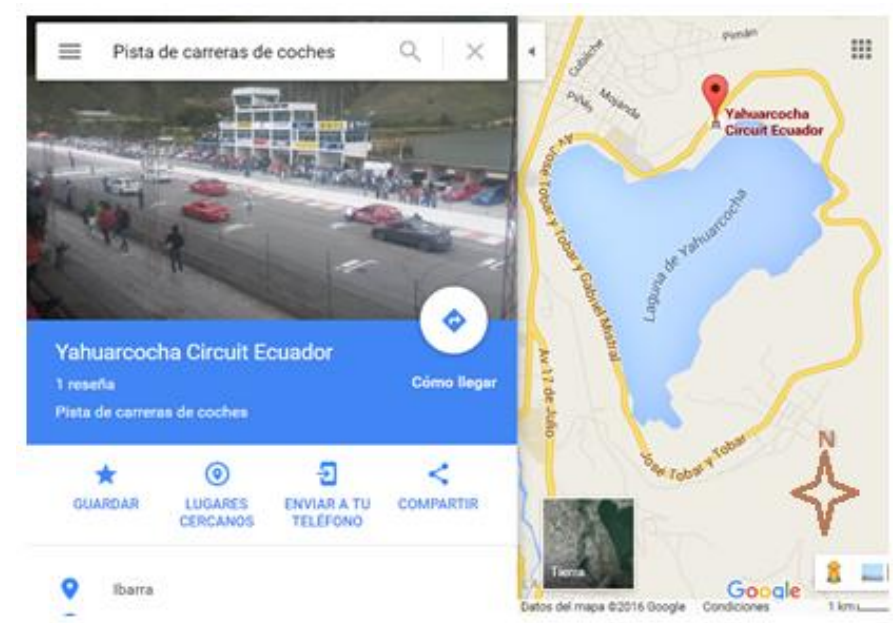

Figura 3. Autódromo de Yaguarcocha. Fuente: Google Maps, 2018

El procedimiento que se siguió para arrancar el motor es el siguiente:

- Comprobar el nivel de aceite

- Comprobar el nivel de agua en el radiador

- Completar el tanque de gasolina hasta el máximo nivel

- Ingresar los valores máximos de combustible en la aplicación Android.

- Poner en marcha el motor

- Dejar el motor unos 3 minutos en marcha de ralentí (800 rpm)

- Acelerar hasta que alcanza la velocidad de funcionamiento, es decir, $50 \mathrm{~km} / \mathrm{h}$ (2 $500 \mathrm{rpm})$ y $90 \mathrm{~km} / \mathrm{h}$ (3 $300 \mathrm{rpm})$.

\subsection{Cálculo del balance térmico}

El balance térmico hace que se cumpla la igualdad:

Energía que ingresa al sistema = Energía que sale del sistema .

Por otro lado, para un proceso con flujo y estado estable se tiene el cumplimiento de la Ecuación 2: 
Donde:

$\Sigma Q$ : sumatoria de calor evacuado al ambiente

$\Sigma H_{\text {salida: }}$ sumatoria de entalpías que salen del sistema

$\Sigma H_{\text {entrada: }}$ sumatoria de entalpías que entran al sistema

Lo que quiere decir que la suma de los calores que atraviesan el sistema es igual a la diferencia entre la suma de entalpías que ingresan al sistema y la suma de entalpías que salen del sistema (Campos, 2015). La variación de entalpía, por su parte, es una medida de la cantidad de energía absorbida o cedida en un sistema termodinámico, es decir, es la variación que se produce por una transformación en la que se puede recibir o aportar energía (como en el presente caso de estudio, energía mecánica), por lo que se puede considerar a la entalpía como numéricamente igual al calor intercambiado con el ambiente (Bernal, 2014).

Para determinar el balance energético, se consideran los siguientes cálculos generales (Ecuaciones 3-9):

Flujo de masa de combustible

$$
\dot{m}_{c}=\rho \frac{V}{\Delta t}
$$

Donde:

$$
\begin{aligned}
& \dot{\mathrm{m}}_{\mathrm{c}}=\text { flujo másico de combustible }[\mathrm{kg} / \mathrm{s}] \\
& \rho=\text { densidad específica de combustible }\left[\mathrm{kg} / \mathrm{m}^{3}\right] \\
& \mathrm{v}=\text { volumen de combustible }\left[\mathrm{m}^{3}\right] \\
& \Delta \mathrm{t}=\text { tiempo de flujo }[\mathrm{s}]
\end{aligned}
$$

Calor liberado por el combustible

$$
\dot{Q}_{c}=\dot{m}_{c} . P C I
$$

Donde:

$$
\begin{aligned}
& \dot{\mathrm{Q}}_{\mathrm{C}}=\text { Calor liberado por el combustible }[\mathrm{kW}] \\
& \mathrm{PCl}=\text { poder calorífico inferior del combustible }[\mathrm{kJ} / \mathrm{kg}]
\end{aligned}
$$

Pérdida de calor por los gases de escape

$$
\dot{Q}_{g}=\dot{m}_{g} \cdot \bar{c}_{p} \cdot\left(T_{e s c}-T_{a m b}\right)
$$

Donde:

$$
\begin{aligned}
& \dot{\mathrm{Q}}_{\mathrm{g}}=\text { calor liberado por los gases de escape }[\mathrm{kJ} / \mathrm{s}] \\
& \dot{\mathrm{m}} \mathrm{g}=\text { flujo másico de los gases }[\mathrm{kg} / \mathrm{s}] \\
& \overline{\mathrm{c}}_{\mathrm{p}}=\text { calor específico a presión constante promedio de los gases } \\
& \mathrm{T}_{\text {esc }}=\text { Temperatura de salida }\left[{ }^{\circ} \mathrm{C}\right] \\
& \mathrm{T}_{\mathrm{amb}}=\text { Temperatura ambiente }\left[{ }^{\circ} \mathrm{C}\right]
\end{aligned}
$$

Flujo de los gases

$$
\dot{m}_{g}=\dot{m}_{a r}+\dot{m}_{\text {comb }}
$$


Donde:

$\dot{\mathrm{m}}_{\mathrm{ar}}=$ flujo de aire $[\mathrm{kg} / \mathrm{s}]$

$\dot{\mathrm{m}}_{\text {comb }}=$ flujo másico de combustible $[\mathrm{kg} / \mathrm{s}]$

Calor perdido en sistema de refrigeración

$$
\dot{Q}_{A}=\dot{m}_{A} \cdot \bar{c}_{A} \cdot\left(T_{s}-T_{e}\right)
$$

Donde:

$\dot{\mathrm{Q}}_{\mathrm{A}}=$ calor en radiador $[\mathrm{kW}]$

$\dot{\mathrm{m}}_{\mathrm{A}}=$ flujo de agua en radiador $[\mathrm{kg} / \mathrm{s}]$

$\overline{\mathrm{c}}_{\mathrm{A}}=$ calor específico de agua $\left[\mathrm{kJ} / \mathrm{kg} .{ }^{\circ} \mathrm{C}\right]$

$\mathrm{T}_{\mathrm{S}}=$ Temperatura de salida del agua $\left[{ }^{\circ} \mathrm{C}\right]$

$\mathrm{T}_{\mathrm{e}}=$ Temperatura de entrada del agua $\left[{ }^{\circ} \mathrm{C}\right]$

\section{Balance energético}

$$
\dot{Q}_{p}=\dot{Q}_{c}-\dot{Q}_{g}-\dot{Q}_{A}-\dot{Q}_{k-c}
$$

Donde:

$\dot{\mathrm{Q}}_{\mathrm{p}=\text { energía útil en el motor }}$

$\dot{\mathrm{Q}}_{\mathrm{C}=\text { calor liberado por el combustible }}$

$\dot{\mathrm{Q}}_{\mathrm{g}=\text { calor de los gases de escape }}$

$\dot{\mathrm{Q}}_{\mathrm{A}}=$ calor del agua de enfriamiento

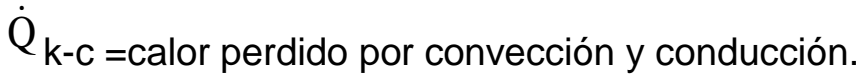

El rendimiento del sistema se determina según la Ecuación 9.

$$
\eta_{\text {energético }}=\frac{Q_{p}}{Q_{c}}
$$

\subsection{Balance exergético (con base en el trabajo disponible)}

La exergía es un parámetro que mide la calidad de la energía en sistemas determinados. No existe una ley de conservación de la exergía, por eso, para realizar un balance de exergía es necesario incluir términos correspondientes a su pérdida (Moore, 2004).

La exergía se define como el trabajo máximo teórico que se puede obtener de un sistema combinado (combinación de un sistema y el ambiente de referencia) cuando el sistema llega al equilibrio (térmico, mecánico y químico) con el ambiente. Este nivel máximo de trabajo proviene de la suma de las contribuciones de exergía termodinámica y química (Sezer y Bilgin, 2013). Estudios como los de Zhu et al. (2014) han determinado que las tasas de destrucción de exergía del proceso de mezcla y el proceso de evaporación ocupan las partes principales de la tasa general de destrucción de exergía. $-15)$

El balance de exergía se determina mediante la siguiente metodología (Ecuaciones 10

Exergía asociada al calor de combustible

$$
E x_{c}=\dot{m}_{c} P C I
$$


Donde:

$E x_{c}=$ Exergía del combustible $[\mathrm{kW}]$

Exergía asociada a los flujos de masa

$$
E x_{f}=\dot{m}\left[h-h_{o}-T_{o}\left(S-S_{o}\right)\right]
$$

Donde:

$E x_{f}=$ exergía del flujo de masa $[\mathrm{kW}]$

$\dot{\mathrm{m}}=$ flujo de masa $[\mathrm{kg} / \mathrm{s}]$

ho $=$ entalpia a $T_{0}$ y $\mathrm{P}_{0}$ en $[\mathrm{kJ} / \mathrm{kg}]$

To $=$ Temperatura Referencia $(298 \mathrm{~K})$

So = entropía a $T_{0}$ y $P_{0}$ en $[\mathrm{kJ} / \mathrm{kg} \mathrm{K}]$

$\mathrm{h}=$ entalpia $[\mathrm{kJ} / \mathrm{kg}]$

$\mathrm{S}=$ entropía $[\mathrm{kJ} / \mathrm{kg} \mathrm{K}]$

El rendimiento exergético se determina a partir de la Ecuación 12.

$$
\eta_{\text {exergética }}=\frac{\sum E_{\text {flujosdeentradas }}-\sum E_{\text {flujosdesdidas }}}{\sum E_{\text {flujosdeentradas }}}
$$

Calor específico a presión constante

El calor específico del aire a una temperatura $T,(K)$, está dado por:

$$
\mathrm{c}_{\mathrm{par}, \mathrm{T}}=\mathrm{C}_{0}+\mathrm{C}_{1} \mathrm{~T}+\mathrm{C}_{2} \mathrm{~T}^{2}+\mathrm{C}_{3} \mathrm{~T}^{3}+\mathrm{C}_{4} \mathrm{~T}^{4} \quad(\mathrm{~J} / \mathrm{kg} \mathrm{K})
$$

Para temperatura entre 200 a $800 \mathrm{~K}$.

Donde:

$$
\begin{aligned}
& C_{0}=+1.0189 E+03 \\
& C_{1}=-1.3784 E-01 \\
& C_{2}=+1.9843 E-04 \\
& C_{3}=+4.2399 E-07 \\
& C_{4}=-3.7632 E-10
\end{aligned}
$$

El calor específico a presión constante del combustible está dado por:

$$
\mathrm{c}_{\mathrm{p} g, \mathrm{~T}}=\mathrm{c}_{\mathrm{par}, \mathrm{T}}+\frac{\mathrm{f}}{1+\mathrm{f}} \theta_{\mathrm{Cp}, \mathrm{T}} \quad(\mathrm{J} / \mathrm{kg} \mathrm{K})
$$

Donde $f=\frac{\dot{m}_{c o m b}}{\dot{m}_{a r}}$

$$
\theta_{\mathrm{Cp}, \mathrm{T}}=\mathrm{CP}_{0}+\mathrm{CP}_{1} \mathrm{~T}+\mathrm{CP}_{2} \mathrm{~T}^{2}+\mathrm{CP}_{3} \mathrm{~T}^{3}+\mathrm{CP}_{4} T^{4}+\mathrm{CP}_{5} \mathrm{~T}^{5}
$$

Para temperatura entre 200 a $800 \mathrm{~K}$

$$
\begin{aligned}
& \mathrm{CP}_{0}=-3.5949 \mathrm{E}+02 \\
& \mathrm{CP}_{1}=+4.5164 \mathrm{E}+00
\end{aligned}
$$




$$
\begin{aligned}
& \mathrm{CP}_{2}=+2.8116 \mathrm{E}-03 \\
& \mathrm{CP}_{3}=-2.1709 \mathrm{E}-05 \\
& \mathrm{CP}_{4}=+2.8689 \mathrm{E}-08 \\
& \mathrm{CP}_{5}=-1.2226 \mathrm{E}-11
\end{aligned}
$$

Los autores Reibán y Ramírez (2014), realizan un estudio similar al propuesto en el presente documento, con la diferencia de que generó datos para diferentes niveles de revoluciones por minuto del motor, con el fin de generar una tabla de datos para un software de la universidad que patrocinó el mencionado estudio. El desarrollo del estudio se realizó utilizando materiales y métodos similares a los que se utilizan en el presente ensayo, donde como principal conclusión se presenta que existe una eficiencia energética transformada en trabajo real de alrededor del $25 \%$, una pérdida de $40 \%$ por gases de escape, $30 \%$ por refrigeración y $5 \%$ por fricción y otras pérdidas. Según Khoobbakht et al. (2016), la tasa total de pérdida de energía del volumen de control incluye todo el calor de pérdidas debidas a la convección de las paredes de la cámara de combustión y la salida entalpía de los gases de escape.

\section{Resultados y Discusión}

\begin{tabular}{|c|c|c|c|c|c|c|c|c|}
\hline \multicolumn{9}{|c|}{ Datos a $50 \mathrm{~km} / \mathrm{h}$ a $2300 \mathrm{rpm}$} \\
\hline \multirow{2}{*}{$\begin{array}{c}\text { Consumo } \\
\left(\mathrm{m}^{3}\right)\end{array}$} & \multirow{2}{*}{$\begin{array}{c}m_{\text {aire }} \\
(\mathbf{k g} / \mathbf{s})\end{array}$} & \multirow{2}{*}{$\begin{array}{l}m_{\text {agua }} \\
(\mathbf{k g} / \mathbf{s})\end{array}$} & \multicolumn{2}{|c|}{ En el Escape } & \multicolumn{2}{|c|}{ En el radiador } & \multirow{2}{*}{$\begin{array}{c}\text { Motor } \\
\begin{array}{c}T_{\text {amb }} \\
(\mathrm{K})\end{array}\end{array}$} & \multirow{2}{*}{$\begin{array}{c}\text { Carter } \\
\begin{array}{c}T_{\text {amb }} \\
(\mathrm{K})\end{array}\end{array}$} \\
\hline & & & $\begin{array}{l}T_{\text {sal }} \\
(\mathrm{K})\end{array}$ & $\begin{array}{l}T_{\text {amb }} \\
(\mathrm{K})\end{array}$ & $\begin{array}{l}T_{\text {ent }} \\
\text { (K) }\end{array}$ & $\begin{array}{l}T_{\text {sal }} \\
(K)\end{array}$ & & \\
\hline 0.0004812 & 0.1 & 0.065 & 362 & 295 & 330 & 358 & 348 & 355 \\
\hline 0.00043 & 0.11 & 0.058 & 368 & 288 & 320 & 339 & 350 & 353 \\
\hline 0.0005 & 0.06 & 0.074 & 350 & 279 & 337 & 372 & 352 & 344 \\
\hline 0.00072 & 0.16 & 0.073 & 359 & 295 & 325 & 360 & 360 & 348 \\
\hline 0.000615 & 0.14 & 0.06 & 360 & 288 & 318 & 350 & 358 & 342 \\
\hline
\end{tabular}

En las Tablas 1 y 2 se muestran los resultados de las 5 mediciones, de los parámetros necesarios para los cálculos del balance térmico del $\mathrm{MCl}$ a $50 \mathrm{~km} / \mathrm{h}$ y $90 \mathrm{~km} / \mathrm{h}$.

\begin{tabular}{|c|c|c|c|c|c|c|c|c|}
\hline \multicolumn{9}{|c|}{ Datos a $90 \mathrm{~km} / \mathrm{h}$ a $3300 \mathrm{rpm}$} \\
\hline \multirow{2}{*}{$\begin{array}{c}\text { Consumo } \\
\qquad\left(\mathrm{m}^{3}\right)\end{array}$} & \multirow[b]{2}{*}{$\begin{array}{c}m_{\text {aire }} \\
(\mathbf{k g} / \mathbf{s})\end{array}$} & \multirow[b]{2}{*}{$\begin{array}{l}m_{\text {agua }} \\
(\mathbf{k g} / \mathbf{s})\end{array}$} & \multicolumn{2}{|c|}{ En el Escape } & \multicolumn{2}{|c|}{ En el radiador } & \multirow{2}{*}{$\begin{array}{c}\text { Motor } \\
T_{\text {amb }} \\
(\mathrm{K})\end{array}$} & \multirow{2}{*}{$\begin{array}{c}\text { Carter } \\
T_{\text {amb }} \\
(\mathrm{K})\end{array}$} \\
\hline & & & $\begin{array}{l}T_{\text {sal }} \\
(\mathrm{K})\end{array}$ & $\begin{array}{l}T_{\text {amb }} \\
(\mathrm{K})\end{array}$ & $\begin{array}{l}T_{\text {ent }} \\
(\mathrm{K})\end{array}$ & $\begin{array}{l}T_{\text {sal }} \\
(\mathrm{K})\end{array}$ & & \\
\hline 0.0011 & 0.3 & 0.085 & 378 & 294 & 335 & 363 & 355 & 364 \\
\hline 0.00109 & 0.28 & 0.078 & 386 & 292 & 334 & 364 & 348 & 368 \\
\hline 0.001121 & 0.36 & 0.088 & 365 & 296.1 & 332 & 361 & 361 & 350 \\
\hline 0.001205 & 0.38 & 0.07 & 376 & 292 & 336 & 360 & 348 & 353 \\
\hline 0.00098 & 0.28 & 0.082 & 373 & 291.65 & 340 & 363 & 353 & 360 \\
\hline
\end{tabular}

Tabla 1. Valores tabulados de la parte experimental a $50 \mathrm{~km} / \mathrm{h}$

Tabla 2. Valores tabulados de la parte experimental a $90 \mathrm{~km} / \mathrm{h}$

En las Tablas 3 y 4 se resumen los parámetros obtenidos al aplicar la metodología anteriormente abordada. 
Tabla 3. Resultado de los valores calculados a $50 \mathrm{~km} / \mathrm{h}$

\begin{tabular}{|c|r|r|r|r|r|r|r|r|}
\hline \multicolumn{10}{|c|}{ Datos a $50 \mathbf{~ k m / h ~ a ~ 2 3 0 0 ~ r p m ~}$} \\
\hline Mediciones & $\begin{array}{c}\mathbf{Q}_{\mathbf{c}} \\
(\mathbf{k J / s})\end{array}$ & $\begin{array}{c}\mathbf{Q}_{\mathbf{g}} \\
(\mathbf{k J / s})\end{array}$ & $\begin{array}{c}\mathbf{Q}_{\mathbf{a}} \\
(\mathbf{k J / s})\end{array}$ & $\begin{array}{c}\mathbf{Q}_{\mathbf{k}} \\
(\mathbf{k J / s})\end{array}$ & $\begin{array}{c}\mathbf{Q}_{\mathbf{p}} \\
(\mathbf{k J / s})\end{array}$ & $\begin{array}{l}\boldsymbol{\eta}_{\text {ener }} \\
(\%)\end{array}$ & $\begin{array}{c}\mathbf{E}_{\mathbf{x c}} \\
(\mathbf{k J / s})\end{array}$ & $\begin{array}{l}\eta_{\text {exe }} \\
(\%)\end{array}$ \\
\hline 1 & 25.37 & 5.66 & 7.61 & 5.07 & 7.02 & 27.69 & 25.37 & 22.42 \\
\hline 2 & 22.67 & 7.42 & 4.61 & 4.53 & 6.10 & 26.92 & 22.67 & 22.63 \\
\hline 3 & 26.37 & 3.61 & 10.83 & 5.27 & 6.64 & 25.20 & 26.37 & 22.00 \\
\hline 4 & 37.97 & 8.64 & 10.69 & 7.59 & 11.04 & 29.07 & 37.97 & 22.31 \\
\hline 5 & 32.43 & 8.51 & 8.03 & 6.48 & 9.40 & 28.99 & 32.43 & 22.35 \\
\hline
\end{tabular}

Nota: Las pérdidas por conducción y convección se ha denominado como $Q_{k}$, considerado en un $20 \%$ de $Q_{c}$, según Reibán \& Ramírez (2014).

Tabla 4. Resultado de los valores calculados a $90 \mathrm{~km} / \mathrm{h}$

\begin{tabular}{|c|c|c|c|c|c|c|c|c|}
\hline \multicolumn{9}{|c|}{ Datos a $90 \mathrm{~km} / \mathrm{h}$ a $3300 \mathrm{rpm}$} \\
\hline Mediciones & $\begin{array}{c}Q c \\
\mathrm{~kJ} / \mathrm{s}\end{array}$ & $\begin{array}{c}\mathrm{Qg} \\
\mathrm{kJ} / \mathrm{s}\end{array}$ & $\begin{array}{c}\mathrm{Qa} \\
\mathrm{kJ} / \mathrm{s}\end{array}$ & $\begin{array}{c}\text { Qk } \\
k J / s\end{array}$ & $\underset{k J / s}{Q p}$ & n ener & $\begin{array}{l}\text { Exc } \\
\mathrm{kJ} / \mathrm{s}\end{array}$ & n exe \\
\hline 1 & 58.01 & 21.26 & 8.12 & 11.60 & 17.02 & 29.35 & 58.01 & 24.84 \\
\hline 2 & 57.48 & 22.21 & 7.98 & 11.49 & 15.79 & 27.47 & 57.48 & 27.18 \\
\hline 3 & 59.12 & 20.91 & 8.70 & 11.82 & 17.67 & 29.89 & 59.12 & 21.04 \\
\hline 4 & 63.55 & 26.91 & 5.73 & 12.71 & 18.19 & 28.62 & 63.55 & 24.26 \\
\hline 5 & 51.68 & 19.21 & 6.43 & 10.33 & 15.69 & 30.37 & 51.68 & 23.38 \\
\hline
\end{tabular}

Como se muestra en la Tabla 3, la eficiencia energética para $50 \mathrm{~km} / \mathrm{h}$ promedió $27.6 \%$ y la exergética de $22.35 \%$. Estos resultados están acordes con los obtenidos por otros estudios como los de Santo (2012), Artés (2012) y Reibán \& Ramírez (2014). El comportamiento a $90 \mathrm{~km} / \mathrm{h}$ se comporta con la misma tendencia tal como se muestra en la Tabla 4, donde la eficiencia energética promedió $29.15 \%$ y la exergética de $24.15 \%$.

El rendimiento de un motor de explosión real puede estar en torno al $25 \%$ o $30 \%$ (Universidad de Sevilla, 2016), donde estudios como los de Catón (2012) en condiciones prácticas avalan el resultado descrito.

Por otro lado, estudio realizado por Yingjian et al. (2014) en el análisis de la eficacia y la pérdida de energía de motores de generación eléctrica de biogás, determinaron que la eficiencia de la generación de energía fue de $28.45 \%$, mientras que la eficiencia de exergía fue de $27.36 \%$.

\subsection{Análisis estadístico}

Con el objetivo de determinar si existe diferencia significativa entre las medias de la eficiencia energética y exergética a 50 y $90 \mathrm{~km} / \mathrm{h}$ respectivamente, es necesario aplicar pruebas de comparación múltiple de medias, para el caso en estudio se aplica la LSD (Least Significant Difference) por medio del software estadístico Statgraphics Centurion XVI.

En la Tabla 5, se muestra el resultado al aplicar la prueba LSD, se puede contractar que tanto para la eficiencia energética y exergética a 50 y $90 \mathrm{~km} / \mathrm{h}$, no poseen diferencia significativa.

Tabla 5. Pruebas de múltiples rangos de los factores

\begin{tabular}{|l|l|l|l|}
\hline & Casos & Media & Grupos Homogéneos \\
\hline Efic.Exerg 50 & 5 & 22.342 & $\mathrm{X}$ \\
\hline Efic.Exerg 90 & 5 & 24.14 & $\mathrm{X}$ \\
\hline Efic.Energ 50 & 5 & 27.574 & $\mathrm{X}$ \\
\hline Efic.Energ 90 & 5 & 29.14 & $\mathrm{X}$ \\
\hline
\end{tabular}

Nota: Método: 95.0 porcentaje LSD 
En la Figura 4, se muestra de forma gráfica la anterior comparación, donde se puede visualizar con mayor exactitud la relación entre la eficiencia energética y exergética, corroborándose que no hay diferencia significativa.

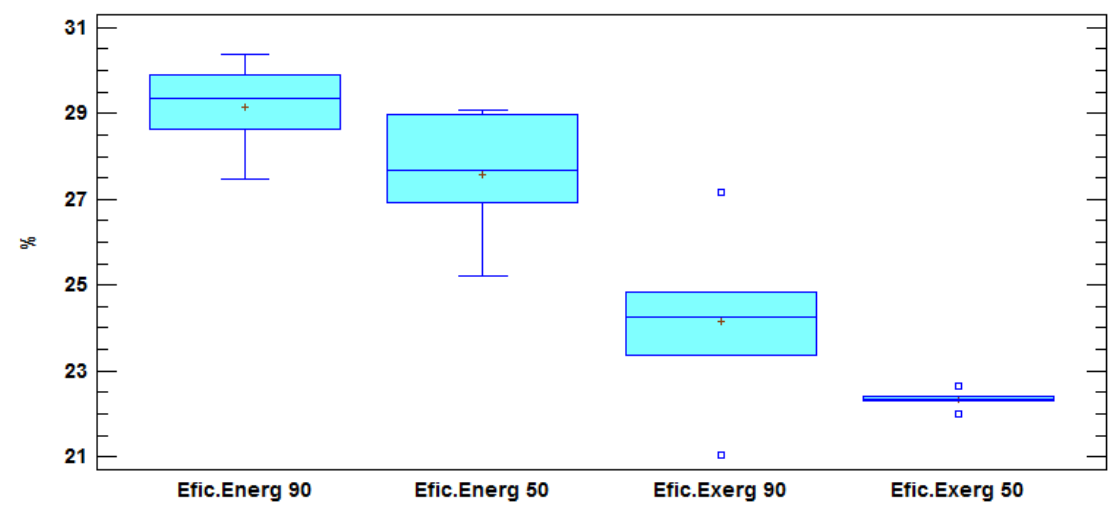

Figura 4: Comparación de las medias para la eficiencia energética y exergética a 50 y $90 \mathrm{~km} / \mathrm{h}$

\subsection{Pérdidas por Calor en el sistema}

Con ayuda de un diagrama de Pareto (Figura 5) se ha evaluado las mayores pérdidas que afectan al sistema, en donde el calor cedido por los gases de escape causa las mayores pérdidas, seguido del calor cedido al sistema de refrigeración con un 32 y $28 \%$ respectivamente, estos resultados concuerdan con los trabajos de Reibán y Ramírez (2014) y Khoobbakht et al. (2016). De forma similar se comporta para $90 \mathrm{~km} / \mathrm{h}$.

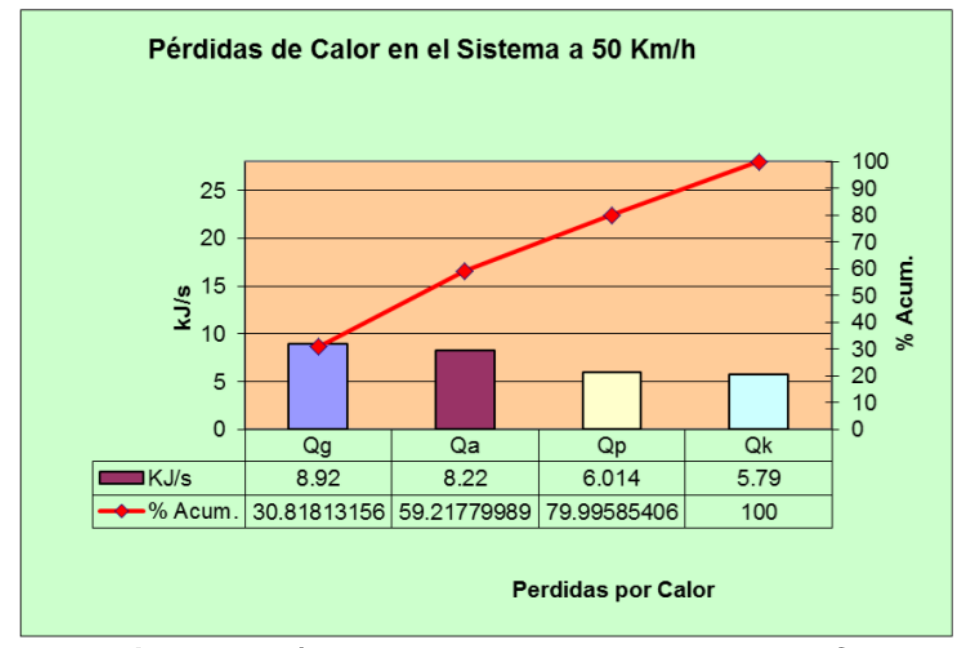

Figura 5. Pérdida de calor en el sistema del $\mathrm{MCl}$

\section{Conclusiones y recomendaciones}

El motor del vehículo de $1.6 \mathrm{~L}$ de cilindrada, que fue utilizado como caso de estudio presenta un rendimiento energético promedio de $27.6 \%$ para una velocidad de $50 \mathrm{~km} / \mathrm{h}$ (2 $300 \mathrm{rpm}$ ). En lo referente a la eficiencia exergética, se determinó que el flujo de exergía promedio del sistema es $22.3 \%$ para el uso del motor a la velocidad de $50 \mathrm{~km} / \mathrm{h}$ (2 $300 \mathrm{rpm}$ ), este comportamiento es similar para la velocidad de $90 \mathrm{~km} / \mathrm{h}$.

Se concluye que entre las velocidades de $50 \mathrm{~km} / \mathrm{h}$ y $90 \mathrm{~km} / \mathrm{h}$ no existe diferencia significativa, ni para la eficiencia energética ni exergética.

A partir de una metodología en la cual se cuantifique la entrada y salida del flujo energético se puede establecer el comportamiento energético y exergético en un $\mathrm{MCl}$. El análisis del Ciclo Otto ha demostrado que, bajo condiciones ideales, un $\mathrm{MCl}$ puede presentar 
una eficiencia de hasta $56.5 \%$, sin embargo, en condiciones reales la eficiencia energética es mucho menor siendo inferior al $30 \%$.

\section{Bibliografía}

Abedin, M. J., Masjuki, H. H., Kalam, M. A., Sanjid, A., Rahman, S. A., \& Masum, B. M. (2013). Energy balance of internal combustion engines using alternative fuels. Renewable and Sustainable Energy Reviews, 26, 20-33.

Andrade, C. (24 de febrero de 2016). Octanaje del Combustible (Gasolina) en Ecuador. Recuperado de http://sinmiedosec.com/octanaje-del-combustible-gasolina-en-ecuador/

Artés, D. (2 de octubre de 2012). Los límites de la eficiencia térmica en motores gasolina y diésel. Obtenido de http://www.diariomotor.com/tecmovia/2012/10/02/los-limites-de-laeficiencia-termica-en-motores-gasolina-y-diesel/

Bernal, C. (2010). Metodología de la Investigación (Tercera Edición ed.)(O. Fernández Palma, Ed.) Bogotá, Colombia.

Bernal, E. (13 de noviembre de 2014). Física termodinámica 6M. Entalpía \& Entropía. Recuperado de: https://estebanbernal10.wordpress.com/tercer-corte/entalpia-entropia/

Campos, P. (22 de octubre de 2015). Instrumentos de Medición para la Evaluación del Consumo de Energía Térmica. Recuperado de https://es.scribd.com/doc/286394459 /unidad-5

Caton, J. (2012). The thermodynamic characteristics of high efficiency, internal-combustion engines. Energy Conversion and Management 58, 84-93.

Cedeño, E. A. L., Rocha-Hoyos, J. C., Zurita, D. B. P., \& Milla, J. C. L. (2018). Evaluación de emisiones de gases en un vehículo liviano a gasolina en condiciones de altura. Caso de estudio Quito, Ecuador. Enfoque UTE, 9(2), 149-158.

Duarte, C. M., Alonso, S., Benito, G., Dachs, J., Montes, C., Pardo Buendía, M., ... \& Valladares, F. (2006). Cambio Global. Impacto de la actividad humana sobre el sistema Tierra. CSIC. Consejo superior de investigaciones científicas.

Erazo, O. (22 de octubre de 2015). Nueva súper generaría más consumo de extra. (D. E. Universo, Entrevistador).

Gaviria Ríos, J. E., Mora Guzmán, J. H., \& Agudelo Santamaría, J. R. (2002). Historia de los motores de combustión interna. Facultad de ingeniería N.26, 68-78.

Grupo FIAT. (2004). Automoción: motores térmicos y sus sistemas auxiliares. México D.F.: Grupo FIAT.

Khoobbakht, G., Akram, A., Karimi, M., \& Najafi, G. (2016). Exergy and energy analysis of combustion of blended levels of biodiesel, ethanol and diesel fuel in a DI diesel engine. Applied Thermal Engineering, 99, 720-729.

Llanes Cedeño, E. A., Zambrano León, V. D., Carvajal, C., Santiago, A., Mena Mena, E. R., \& Rocha Hoyos, J. C. (2017). Teoría de Selección y Dimensionamiento del Parque Automotor. Recuperado de: http://repositorio.espe.edu.ec/xmlui/handle/21000/13755

López Terán, J. L. (2013). Evaluación del consumo de combustible de vehículos livianos en el Distrito Metropolitano De Quito (Doctoral dissertation, Quito: EPN, 2013).

Moore, R. (2004). Procesos de interacción de redes energéticas. Sydney, Australia: Mathematics Department, Macquarie University.

Piedrahita, C. A. R. (2009). Contribución al conocimiento del comportamiento térmico y la gestión térmica de los motores de combustión interna alternativos (Doctoral dissertation). Recuperado de: https://riunet.upv.es/bitstream/handle/10251/4923 /tesisUPV3034.pdf.

Reibán Heredia, J. M., \& Ramírez Velásquez, C. A. (2014). Análisis del balance energético e implementación de un banco didáctico con visualización de datos en tiempo real en un motor Toyota 2B diesel para el Laboratorio de Ingeniería Automotriz (Bachelor's thesis).

Rocha-Hoyos, J. C., L. E. Tipanluisa, V. D. Zambrano y A. A. Portilla, en prensa (2018), Estudio de un Motor a Gasolina en Condiciones de Altura con Mezclas de Aditivo Orgánico en el Combustible, Inf. Tecnol., 28(2). 
Santo, D. E. (2012). Energy and exergy efficiency of a building internal combustion engine trigeneration system under two different operational strategies. Energy and Buildings, 53, 28-38.

Segura, L. M. S., \& Arriaga, J. A. L. (2003). Principios básicos de contaminación ambiental. UAEM.

Sezer, İ., \& Bilgin, A. (2013). Effects of charge properties on exergy balance in spark ignition engines. Fuel, 112, 523-530.

Universidad de Sevilla. (18 de abril de 2016). Ciclo Otto (GIE). Obtenido de Departamento de Física Aplicada: http://laplace.us.es/wiki/index.php/Ciclo_Otto_(GIE)

Vásquez, M., Lugo, C., \& Gómez, C. (2004). Historia Universal 2. México D.F.: Limusa.

Yingjian, L., Qi, Q., Xiangzhu, H., \& Jiezhi, L. (2014). Energy balance and efficiency analysis for power generation in internal combustion engine sets using biogas. Sustainable Energy Technologies and Assessments 6, 25-33.

Zhu, S., Deng, K., \& Qu, S. (2014). Thermodynamic analysis of an in-cylinder waste heat recovery system for internal combustion engines. Energy 67, 548-556. 